\title{
Kann die Saatgutbehandlung mit dem Safener Cyprosulfamid die Verträglichkeit von Isoxaflutole in Mais steigern, die Unkrautbekämpfung verbessern und die Wirkstoffmenge verringern?
}

\author{
Roland Gerhards $^{1}$ (D) $\cdot$ Hans-Joachim Santel ${ }^{1}$ \\ Eingegangen: 10. Juni 2020 / Angenommen: 11. September 2020 / Online publiziert: 8. Oktober 2020 \\ (c) Der/die Autor(en) 2020
}

\section{Zusammenfassung}

Herbizide Wirkstoffe werden in kommerziellen Produkten manchmal mit Safenern kombiniert, um die Verträglichkeit des Herbizids für die Kulturpflanze zu gewährleisten. Dabei regen Safener meist die Metabolisierung von Herbiziden in der Kulturpflanze an. Das Herbizid Isoxaflutole (IFT) wird in Mais mit dem Safener Cyprosulfamid (CSA) als Co-Formulierung angewendet. In dieser Arbeit wurde untersucht, ob CSA auch zeitlich getrennt von IFT als Saatgutbehandlung den Mais vor Herbizidschäden durch IFT schützen kann und ob die Unkrautbekämpfung in diesem Fall höher ist als bei der Co-Formulierung, weil die Unkräuter durch die Saatbehandlung nicht mit CSA in Kontakt kommen.

Es wurden von 2016-2018 insgesamt fünf Topfversuche als randomisierte, vollständige Blockanlage mit 15 Wiederholungen durchgeführt. Dabei wurde IFT ohne CSA, mit CSA als Co-Formulierung und mit CSA-Beize in der einfachen und doppelten Feldaufwandmenge im 2-Blatt-Stadium von Mais appliziert und 28 Tage nach der Applikation die Herbizidschäden an Mais und Unkräutern bonitiert und die Sprosslänge und Trockenmasse der Maisstängel gemessen.

In allen Versuchen reagierte Mais sensibel mit Bleichungen an den Blättern auf IFT mit bis zu $62 \%$ Schäden. Die Kombination von IFT und CSA reduzierte den vom Herbizid verursachten Schaden signifikant. Die schützende Wirkung von CSA war stärker, wenn der Safener simultan mit dem Herbzid gespritzt wurde (5-10\% Schaden) als bei Beizanwendung (15-25\% Schaden). Im Mittel aller Versuche war die Sprosslänge des mit der der Co-Formulierung von IFT und CSA behandelten Maises auf dem Niveau der unbehandelten Kontrolle und höher als in den IFT-Behandlungen. Die Beizvariante mit $100 \mathrm{~g} / \mathrm{ha} \mathrm{IFT} \mathrm{zeigte} \mathrm{die} \mathrm{längsten} \mathrm{Sprosse.} \mathrm{Der} \mathrm{Safener} \mathrm{konnte} \mathrm{die} \mathrm{herbizidbedingte} \mathrm{Reduktion} \mathrm{der} \mathrm{Trockenmassebildung}$ nur teilweise verhindern. Die Behandlungen mit IFT ohne Zusatz des Safeners zeigten die niedrigsten Sprossmassen der Maisstängel. Die Bekämpfung von Amaranthus retroflexus, Echinochloa crus-galli und Setaria viridis gelang in allen Behandlungen zu $100 \%$. Nur gegen Stellaria media war die Bekämpfung ohne Safener besser als mit CSA-Beize (92\% Bekämpfungsrate) und Co-Formulierung (79\%).

Mit der Saatgutbeizung können ca. 80\% des Safeners gegenüber der Co-Formulierung eingespart werden.

Schlüsselwörter Formulierung $\cdot$ Selektivität $\cdot$ Herbizidmetabolisierung $\cdot$ Safening $\cdot$ Beizung

Roland Gerhards

gerhards@uni-hohenheim.de

1 Fachgebiet Herbologie, Universität Hohenheim,

70593 Stuttgart, Deutschland 


\title{
Can Seed Treatment of the Safener Cyprosulfamid Increase Maize Tolerance to the Herbicide Isoxaflutole, Improve Weed Control Efficacy and Reduce Application Rate of Cyprosulfamid?
}

\begin{abstract}
Herbicdes are sometimes combined with safeners in commercial products to increase crop tolerance. Safeners usually induce metabolism of herbicides in the crop. Isoxaflutole (IFT) is a herbicide used in maize in combination with the safener cyprosulfamid (CSA) as co-formulation. In this study, it was investigated if CSA can also be used as seed treatment with temporal variation to IFT application and if seed treatment provides equal crop safening than the co-formulation. We expected that weed control efficacy would be higher as seed treatment because weeds would not get in contact to CSA. Five pot experiments were carried out from 2016 until 2018. All experiments were realized as randomized complete block design with 15 repetitions. IFT was applied without CSA, as co-formulation mixed with CSA and seperately from CSA (seed treatment) in the full and double recommended field dose when maize had developed two leaves. Assessments of crop damage, crop heigth, crop biomass and weed control efficacy were made 28 days after IFT treatment.

In all experiments, maize responded extremely sensitive to IFT with bleaching symptoms and crop failure of up to $62 \%$. The combinations of IFT and CSA significantly reduced crop damage. Crop safening was stronger in the co-formulation with only $5-10 \%$ crop damage than in the combination of seed treatment of CSA and postemergent application of IFT with 15-25\% damage. Averaged over all five experiments, crop height of the co-formulation was equal to the untreated control plots and the seed treatment with $100 \mathrm{~g} / \mathrm{ha}$ IFT resulted even in higher maize stems. However, maize dry biomass was partly lower in all IFT treatments compared to the untreated control. Nevertheless, crop safening significantly increased maize biomass compared to IFT alone. Control efficacy against Amaranthus retroflexus, Echinochloa crus-galli and Setaria viridis was $100 \%$ in all treatments. Only, efficacy against Stellaria media was reduced to $92 \%$ with the seed treatment and $79 \%$ with the co-formulation. CSA rate was $80 \%$ lower in the seed treatment compared to the co-formulation.
\end{abstract}

Keywords Formulation $\cdot$ Selectivity $\cdot$ Herbicide metabolism $\cdot$ Safening $\cdot$ Seed treatment

\section{Einleitung}

Safener, auch Antidote genannt, sind synthetisch hergestellte Substanzen, die Pflanzen vor Herbizidschäden schützen (Hatzios und Hoagland 1989). Der praktische Nutzen ist ein mehrfacher. Beispielsweise erlaubt der Safener Cyprosulfamid die Ausdehnung des Anwendungsfensters des Herbizids Isoxaflutole in Mais vom reinen Vorauflauf bis in den frühen Nachauflauf (3-Blatt-Stadium) der Kultur. Der Zusatz von Mefenpyr zu Fenoxaprop ermöglicht die Anwendung in Gerste. Die verbesserte Kulturverträglichkeit unter Safenereinfluss kann eine Erhöhung der Herbiziddosis erlauben und damit eine größere Breite und höhere Sicherheit der Herbizidwirkung zur Folge haben. Ebenfalls wird die „Elastizität“ der Verträglichkeit von Herbiziden unter ungünstigen Umweltbedingungen (Temperatur, variable Bodenqualität) verbessert. Safener tragen indirekt auch zur Vermeidung der Herbizidresistenz bei, weil sie die Zahl der verfügbaren Wirkstoffe/Wirkmechanismen in einer Kulturpflanze erhöhen und damit den Selektionsdruck auf einen einzelnen Wirkstoff/Wirkmechanismus verringern. Die durch Safener erweiterte Wirkstoffauswahl bietet dem Anwender mehr Optionen zur Rotation von Wirkstoffen.

Bislang sind nur Safener für monokotyle Kulturpflanzen bekannt. Für dikotyle Kulturpflanzen sind bisher keine kommerziell nutzbaren Safener verfügbar (Davies und Ca- seley 1999). Die Spezifität für die Kultur, den Wirkstoff oder den molekularen Wirkungsmechanismus variiert von Safener zu Safener (Tab. 1). Es gibt feste „Wirkstoff-Safener-Paare" aber es gibt auch Safener, welche die Verträglichkeit von mehreren Wirkstoffen mit gleichen oder unterschiedlichen Wirkungsmechanismen für eine oder mehrere Arten von Kulturpflanzen verbessern können.

Üblicherweise werden Safeners mit dem oder den herbiziden Wirkstoffen in einem Produkt formuliert und ausgebracht. Nach Herstellen der Spritzbrühe werden die Wirkstoffe und der Safener gemeinsam, d.h. simultan, entweder auf den Boden im Vorauflauf- oder auf das Blatt im Nachauflaufverfahren appliziert. Es ist davon auszugehen, dass die simultane Applikation nicht notwendigerweise eine gleichzeitige Aufnahme von Herbizid und Safener zur Folge hat. Jede Komponente bewegt sich auf der Basis ihrer eigenen physikalisch-chemischen Eigenschaften unabhängig von den Mischpartnern im Boden oder vom Ort der Retention zum Ort der Wirkung.

Die Schutzwirkung der Safener beruht auf der Intensivierung der Metabolisierung von Herbiziden in den Kulturpflanzen. Die Erhöhung der Metabolisierungsrate hält die Herbizidkonzentration in der Kulturpflanze und die Dauer der Einwirkung auf die Nutzpflanze unter der für die Entstehung von Pflanzenschäden kritischen Schwelle. Die Stimulierung der Metabolisierung geschieht auf mehreren Ebenen (Riechers et al. 2010). Der eingesetzte Safener wirkt dabei 
Tab. 1 Ausgewählte Beispiele von kommerziell erfolgreichen Kombinationen aus Herbizid und Safener (modifiziert nach Gerhards und Santel 2017)

\begin{tabular}{llll}
\hline Safener & Herbizid & Wirkungort Herbizd & Zielkulturen \\
\hline Benoxacor & S-Metolachlor & VLCFA-Synthase & Mais, Sorghum \\
Cloquintocetmexyl & Clodinafop & Acetyl-CoA Synthase & Weizen \\
Cloquintocetmexyl & Pinoxaden & Acetyl-CoA Synthase & Weizen, Gerste \\
Cyprosulfamid & Isoxaflutole & 4-HPPD & Mais \\
Cyprosulfamid & Thiencarbazone & Acetolactat-Synthase & Mais \\
& Foramsulfuron & & \\
& Iodosulfuron & & Reis \\
Fenclorim & Pretilachlor & VLCFA-Synthase & Mais \\
Isoxadifen & Foramsulfuron & Acetolactat-Synthase & Mais \\
Isoxadifen & Tembotrione & 4-HPPD & Weizen, Gerste \\
Mefenpyr & Fenoxaprop & Acetyl-CoA Synthase & Weizen, Roggen, Triticale, \\
Mefenpyr & Mesosulfuron & Acetolactat-Synthase & Dinkel \\
& Iodosulfuron & &
\end{tabular}

als Signal und reagiert nicht selbst mit dem abzubauenden Herbizid. Es ist also kein stöchiometrisches Verhältnis zwischen der Anzahl der Wirkstoffmoleküle und der Anzahl der Safenermoleküle für die Schutzwirkung erforderlich.

Die Metabolisierung von Herbiziden findet über eine Sequenz von Reaktionsstufen statt (Gerhards und Santel 2017). Auf der ersten Stufe wird das Substitutionsmuster des Wirkstoffs oder seine Grundstuktur durch Spaltung so verändert, dass die biologische Wirkung verloren geht. Das geschieht beispielsweise durch Oxidationen mithilfe von Cytochrom-450 Oxygenasen, Esterhydrolysen mittels Hydrolasen oder oxidativer Desaminierung. Der Wirkstoff wird in der ersten Phase (Konversion) zu unwirksamen Metaboliten abgebaut. In der zweiten Phase (Konjugation) der Metabolisierung werden die Abbauprodukte aus Phase 1 mit zelleigenen Komponenten wie Glutathion oder aktivierten Zuckermolekülen verbunden. Dabei ändert sich die Löslichkeit, da lipophile Metaboliten zu hydrophilen Konjugaten aus Metabolit und polarer Komponente gekoppelt werden. In Phase 3 werden die Konjugate in andere Zellkompartimente, wie beispielsweise die Vakuole, transportiert und dabei weiter abgebaut oder zusätzlich konjugiert, bevor die nicht weiter verwertbaren Produkte der vorangegangenen Abbaureaktionen in einer vierten Abbauphase als gebundene Rückstände in die Zellwandstruktur eingebaut werden. Die stimulierende Wirkung von Safenern auf den Herbizidabbau beschränkt sich nicht auf die Bereitstellung der für die Herbizidmetabolisierung benötigten zellulären Produkte wie Glutathion. Sie umfasst auch die Erhöhung der Aktivität der beteiligten bereits vorhandenen Enzymmoleküle und die zusätzliche Neusynthese von solchen Enzymmolekülen. Safener erhöhen damit beides, die Aktivität des Herbizidabbaus und die Kapazität für Herbizdabbau. Sie wirken also auf bereits vorhandene Komponenten und sind zusätzlich in der Lage, die Expression bestimmter Gene zu induzieren. Der Weg der Metabolisierung eines Herbizids wird durch einen Safener nicht verändert. Es ent- stehen keine neuen oder zusätzlichen Metabolite. Nur die Abbaugeschwindigkeit wird erhöht (Kraehmer et al. 2014; Rosinger 2014).

Pflanzen nutzen für die Herbizidmetabolisierung Enzymsysteme, Reaktionssequenzen und Mechanismen, die im Laufe der Evolution als Schutz gegen die Wirkung toxischer Stoffe (organische Komponenten, Schwermetall) und biotischen oder abiotischen Stress entstanden. Es ist daher nicht verwunderlich, dass nach Anwendung von Mischungen aus Herbiziden und Safenern gelegentlich über positive Safenereffekte in Situationen berichtet wird, in denen die Kulturpflanzen zeitweise unter Umweltstress leiden (Gerhards und Santel 2017).

Safener reduzieren zwar das Schadensrisiko von Herbiziden beträchtlich, können aber auch ganz oder teilweise versagen. Unter Bedingungen, unter denen Herbizid und Safener durch unterschiedliche Wanderung oder Transport räumlich und damit zeitlich zu weit getrennt oder verdünnt werden, kann die erreichbare Safenerkonzentration am Ort der Wirkung zu niedrig werden. Die erforderliche Schutzwirkung bleibt dann aus (Gerhards und Santel 2017).

Ziel dieser Studie war es, den Safener Cyprosulfamid (CSA) als Saatgutbeize nur dem Mais bereitzustellen und das Herbizid Isoxaflutole (IFT) allein ganzflächig auszubringen. Damit sollte verhindert werden, dass Unkräuter mit dem Safener in Kontakt kommen und möglicherweise durch den Safener geschützt werden. Eine Begrenzung der mit Safener behandelten Fläche auf die Oberfläche der Maiskörner soll die Aufwandmenge von CSA deutlich vermindern und den Eintrag von Chemikalien in die Umwelt reduzieren. Es sollte geprüft werden, ob der Mais durch die Saatgutbeize ähnlich gut vor Schäden durch IFT bewahrt wird wie durch die simultane Applikation der Co-Formulierung von IFT und CSA. Weiterhin sollte untersucht werden, ob Unkräuter mit der getrennten Ausbringung von CSA als Beize und IFT als Nachauflaufbehandlung besser $\mathrm{zu}$ bekämpfen sind. 
Tab. 2 Behandlungen der Pflanzen mit Isoxaflutole in unterschiedlichen Formulierungen

\begin{tabular}{|c|c|c|}
\hline Varianten & Formulierung & Aufwandmengen, Wirkstoff \\
\hline Kontrolle & Wasser & $200 \mathrm{~L} \mathrm{ha}^{-1}$ \\
\hline Balance Pro ${ }^{\circledR}$, Bayer CropScience & $240 \mathrm{SC}$ (Suspensionskonzentrat) mit $240 \mathrm{~g} / 1$ Isoxaflutole (IFT) & $100 \mathrm{~g} \mathrm{ha}^{-1} \mathrm{IFT}$ \\
\hline Balance Pro ${ }^{\circledR}$ & $240 \mathrm{SC}$ & $200 \mathrm{~g} \mathrm{ha}^{-1} \mathrm{IFT}$ \\
\hline Balance ${ }^{\circledR}$ flexx, Bayer CropScience & $240 \mathrm{SC}+240 \mathrm{mit} 240 \mathrm{~g} / \mathrm{l} \mathrm{IFT}+240 \mathrm{~g} / \mathrm{l}$ Cyprosulfamid (CSA) & $100 \mathrm{~g} \mathrm{ha}^{-1} \mathrm{IFT}+100 \mathrm{~g} \mathrm{ha}^{-1} \mathrm{CSA}$ \\
\hline Balance ${ }^{\circledR}$ flexx & $240 \mathrm{SC}+240$ & $200 \mathrm{~g} \mathrm{ha}^{-1} \mathrm{IFT}+200 \mathrm{~g} \mathrm{ha}^{-1} \mathrm{CSA}$ \\
\hline Balance Pro ${ }^{\circledR}$ & $240 \mathrm{SC}$ & $100 \mathrm{~g} \mathrm{ha}^{-1} \mathrm{IFT}+2 \mathrm{~g} \mathrm{ha}^{-1} \mathrm{CSA}$ \\
\hline Balance Pro ${ }^{\circledR}$ & $240 \mathrm{SC}$ & $200 \mathrm{~g} \mathrm{ha}^{-1} \mathrm{IFT}+2 \mathrm{~g} \mathrm{ha}^{-1} \mathrm{CSA}$ \\
\hline
\end{tabular}

Tab. 3 Boniturskala mit Erklärungen zur Bewertung des Schadens [\%] (Kraehmer et al. 2014)

\begin{tabular}{|c|c|}
\hline Boniturskala & Symptome \\
\hline 0 & $\begin{array}{l}\text { Gesunde Pflanze, stabiler Stängel, keine Ausbleichung, keine Chlorosen oder Nekrosen, hohe Biomasseproduktion und } \\
\text { Wuchshöhe }\end{array}$ \\
\hline $10-30$ & $\begin{array}{l}\text { Schädigung bis zum 3-Blatt-Stadium, auf dem ersten Blatt in der Mitte chlorotischer Streifen, auf folgenden zwei Blättern } \\
\text { leichte Chlorosen am Blattrand, je nach Ausprägung niedrigere oder höhere Bewertung, Wuchshöhe leicht vermindert }\end{array}$ \\
\hline $40-60$ & $\begin{array}{l}\text { Chlorosen fleckig auf den ersten drei bis vier Blättern verteilt, mittlere Wuchshöhe und Biomasseproduktion, leichte Abnah- } \\
\text { me der Standfestigkeit }\end{array}$ \\
\hline $70-80$ & $\begin{array}{l}\text { Übergang auf den Blättern von chlorotischem zu ausgebleichtem Gewebe, Wuchs und Biomasseproduktion stark einge- } \\
\text { schränkt, mittlere Abnahme der Standfestigkeit }\end{array}$ \\
\hline $80-90$ & $\begin{array}{l}\text { Beginnende Nekrosen an den Blattspitzen, komplette Ausbleichung der Pflanze, nur noch wenige grüne Streifen im Gewebe, } \\
\text { Wachstum ab 2-3-Blatt-Stadium eingestellt, starke Abnahme der Standfestigkeit }\end{array}$ \\
\hline 100 & Pflanze komplett ausgebleicht, nekrotisch, abgestorbene Pflanze, Wachstum ab dem 2-3-Blatt-Stadium eingestellt \\
\hline
\end{tabular}

\section{Material und Methoden}

\section{Versuchsaufbau}

Es wurden 2016 und 2017 zwei Versuche im Gewächshaus des Instituts für Phytomedizin der Universität Hohenheim unter kontrollierten Bedingungen bei $25^{\circ} \mathrm{C}$ Tag- und $15^{\circ} \mathrm{C}$ Nachttemperatur, $12 \mathrm{~h}$ Beleuchtung und $12 \mathrm{~h}$ ohne Beleuchtung und täglicher Bewässerung auf die Pflanztöpfe angelegt. Drei weitere Versuche wurden 2018 in der Vegetationsanlage des Instituts für Phytomedizin der Universität Hohenheim unter freilandähnlichen Bedingungen und Bewässerung nach Bedarf durchgeführt. Die Versuche wurden als randomisierte vollständige Blockanlage mit 15 Wiederholungen realisiert.

\section{Pflanzenanzucht}

Einen Tag vor der Aussaat wurden die Maissamen der Sorte Danubio gebeizt und dann in gut bewässerte Töpfe $(11 \mathrm{~cm} \times 11 \mathrm{~cm} \times 12 \mathrm{~cm})$ ausgesät. Die Aussaattiefe betrug $3 \mathrm{~cm}$. Es wurde eine Maispflanze je Topf gesät. Das Bodensubstrat bestand aus 50\% Sand, 25\% Lehm und 25\% Komposterde. Der pH-Wert betrug 7,1.

$\mathrm{Zu}$ Zeitpunkt des Auflaufen der Maispflanzen wurden jeweils drei Unkräuter der Arten Echninochloa crus-galli (L.) P. Beauv. (Hühnerhirse), Setaria viridis (L.) P. Beauv. (Grüne Borstenhirse), Amaranthus retroflexus L. (Zurückgebogener Amarant) und Stellaria media (L.) Vill. (Vogelmiere) in die Pflanztöpfe pikiert. Die Unkräuter befanden sich zu diesem Zeitpunkt ebenfalls im Keimblattstadium.

\section{Beizung der Maissamen mit dem Safener}

Vor der Beizung wurden zu große, zu kleine und beschädigte Maissamen aussortiert. Die homogene Charge garantierte, dass jeder Samen ein gleiches Rotationsverhalten im Beizgefäß zeigte und mit der gleichen Menge an Beize bedeckt wurde. Pro Beizvorgang wurden 50 Samen behandelt. Das Beizgefäß war ein 150-ml-Becherglas aus Duranglas. Die drei Bestandteile der Beizmischung wurden in der Reihenfolge Formulierhilfsstoff (FHS), Safener (CSA) und deionisiertes Wasser in 1,5-ml-Eppendorfgefäße pipettiert und anschließend auf dem Vortexschüttler $10 \mathrm{~s}$ gemischt. Das Beizvolumen der einzelnen Safenervarianten betrug $120 \mu l$ pro Beizvorgang. Das Volumen wurde auf $200 \mu \mathrm{l} \mathrm{er-}$ höht, um das Pipettieren aus den Eppendorfgefäßen zu erleichtern. Die Anzahl der Umdrehungen des Schüttlers betrug 2420-2680 UpM. Der Mais wurde im Becherglas vor der Zugabe der Beize geschüttelt. Der Beizvorgang startete mit dem Pipettieren der Beize in das Becherglas und dauerte $30 \mathrm{~s}$. Danach wurde die Öffnung des Becherglases mit der Handfläche bedeckt und das Gefäß leicht auf den Vortexschüttler gedrückt, um dadurch eine optimale Rotation der Samen sicherzustellen. 

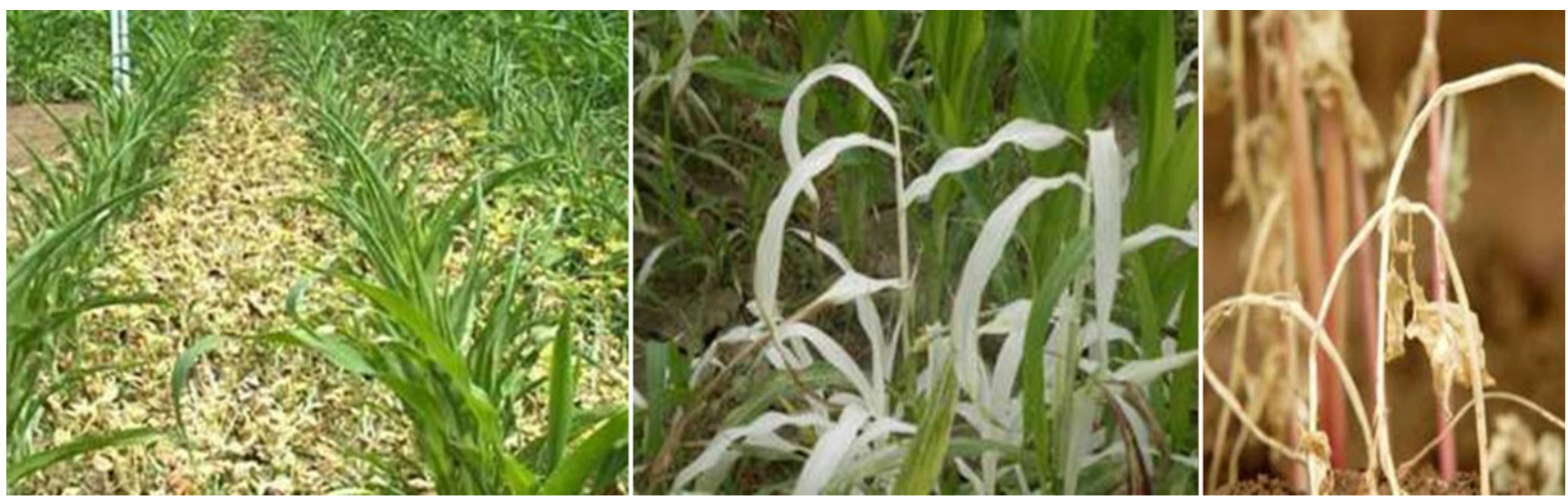

Abb. 1 Wirkungssymptome nach Anwendung eines Herbizids, das die Biosynthese der Carotionoide hemmt. Gut erkennbar sind (von links nach rechts) die selektive Wirkung, hier in Mais, die schneeweiße Färbung neuer Gewebe und Organe, die gelegentlich auftretende sekundäre Rotfärbung und das mit dem Absterben verbundene Verbräunen der Pflanzen (Gerhards und Santel 2017)

\section{Herbizidapplikationen}

Die getesteten Varianten beinhalteten eine Kontrolle (C), in der die Pflanzen nur mit Wasser behandelt wurden und sechs Behandlungen mit IFT in der empfohlenen Feldaufwandmenge von $100 \mathrm{~g} \mathrm{ha}^{-1}$ und in der doppelten Aufwandmenge von $200 \mathrm{~g} \mathrm{ha}^{-1}$ im Nachauflauf, als die Maispflanzen zwei Laubblätter (BBCH 12) ausgebildet hatten. Diese beiden Behandlungen wurden allein (I100, I200), in CoFormulierung mit $100 \mathrm{~g} \mathrm{ha}^{-1}$ bzw. $200 \mathrm{~g} \mathrm{ha}^{-1}$ CSA (IC100, IC200) und getrennt nach Saatgutbehandlung mit $2 \mathrm{~g} \mathrm{ha}^{-1}$ CSA appliziert (Tab. 2). IFT hemmt die Carotinoidbiosynthese. Carotinoide sind Pflanzenpigmente von gelber bis roter Farbe (Ahrens et al. 2013). Sie dienen als akzessorische Pigmente in der Photosynthese. Gleichzeitig sind Carotinoide „Schirmpigmente“, die Chlorophyll bei hoher Einstrahlung vor dauernder Photooxidation durch „Überbelichtung" und oxidativem Stress durch aktivierten Sauerstoff schützen (Hamprecht und Witschel 2012). IFT gehört zur chemischen Klasse der Triketone, die innerhalb der Carotinoidbiosynthese das Enzym 4-Hydroxyphenylpyruvat-Dioxygenase (4-HPPD) inhibieren (Hawkes 2012). Es kann im Vor- und Nachauflauf eingesetzt werden. Die Behandlung mit IGT führt zum Ausfall der Bildung gelber bis roter fettlöslicher Pigmente und zur Photooxidation der vorhandenen Chlorophylle. Die betroffenen Pflanzen oder Pflanzenteile werden weitgehend entfärbt und erscheinen (schnee)weiß. Aus diesem Grund werden derartig wirkende Herbizide auch als Bleichmittel (bleacher) bezeichnet (Abb. 1).

Die Applikation von IFT und CSA erfolgte in einer computergesteuerten Spritzkabine mit einer Flachstrahldüse (Lechler ID8002). Der Spritzstand war kalibriert für eine Aufwandmenge von 200 $\mathrm{L} \mathrm{ha}^{-1}$ bei einer Geschwindigkeit von $800 \mathrm{~mm} \mathrm{~s}^{-1}$ und einer Höhe der Düse von $500 \mathrm{~mm}$ über den Pflanzen und einem Spritzdruck von $300 \mathrm{kPa}$.

\section{Messungen}

Jeweils 14 und 28 Tage nach der Herbizidapplikation wurden die visuellen Schäden an den Maispflanzen und den Unkräutern im Vergleich zur unbehandelten Kontrolle bonitiert. Hierzu wurde die in Tab. 3 dargestellte Boniturskala verwendet, die nach den Richtlinien der EPPO drei Schadwirkungen beinhaltet: 1) Anzahl der abgestorbenen Pflanzen, 2) Wachstumsdepressionen und 3) Wachstumsanomalien (Verfärbungen und Verkrümmungen). Aus diesen drei Schadwirkungen wurde ein Boniturwert geschätzt.

Außerdem wurde die Höhe der Maispflanzen mit einem Meterstab gemessen. Nach 28 Tagen wurden die Pflanzen kurz über dem Boden abgeschnitten, $48 \mathrm{~h}$ bei $80^{\circ} \mathrm{C}$ getrocknet und anschließend gewogen.

\section{Ergebnisse}

\section{Schädigung der Maispflanzen}

In allen durchgeführten Versuchen reagierte Mais sensibel auf IFT, das auf die Blätter appliziert wurde mit bis zu $62 \%$ Schädigung. In der Aufwandmenge von $200 \mathrm{~g} \mathrm{ha}^{-1}$ waren die Maispflanzen stärker gebleicht und mehr Pflanzen abgestorben als bei der empfohlenen Feldaufwandmenge von $100 \mathrm{~g} \mathrm{ha}^{-1}$ (Abb. 1 und 2). Die Kombination von IFT und CSA reduzierte den vom Herbizid verursachten Schaden signifikant. Die schützende Wirkung von CSA war stärker, wenn der Safener simultan mit dem Herbzid gespritzt wurde als bei Beizanwendung. Hier wurden nur noch $5-10 \%$ der Pflanzen geschädigt. Nach Beizung waren die Schadsymptome zwar schwächer als in der reinen IFT-Behandlung aber mit 15-25\% höher als in der Co-Formulierung. 

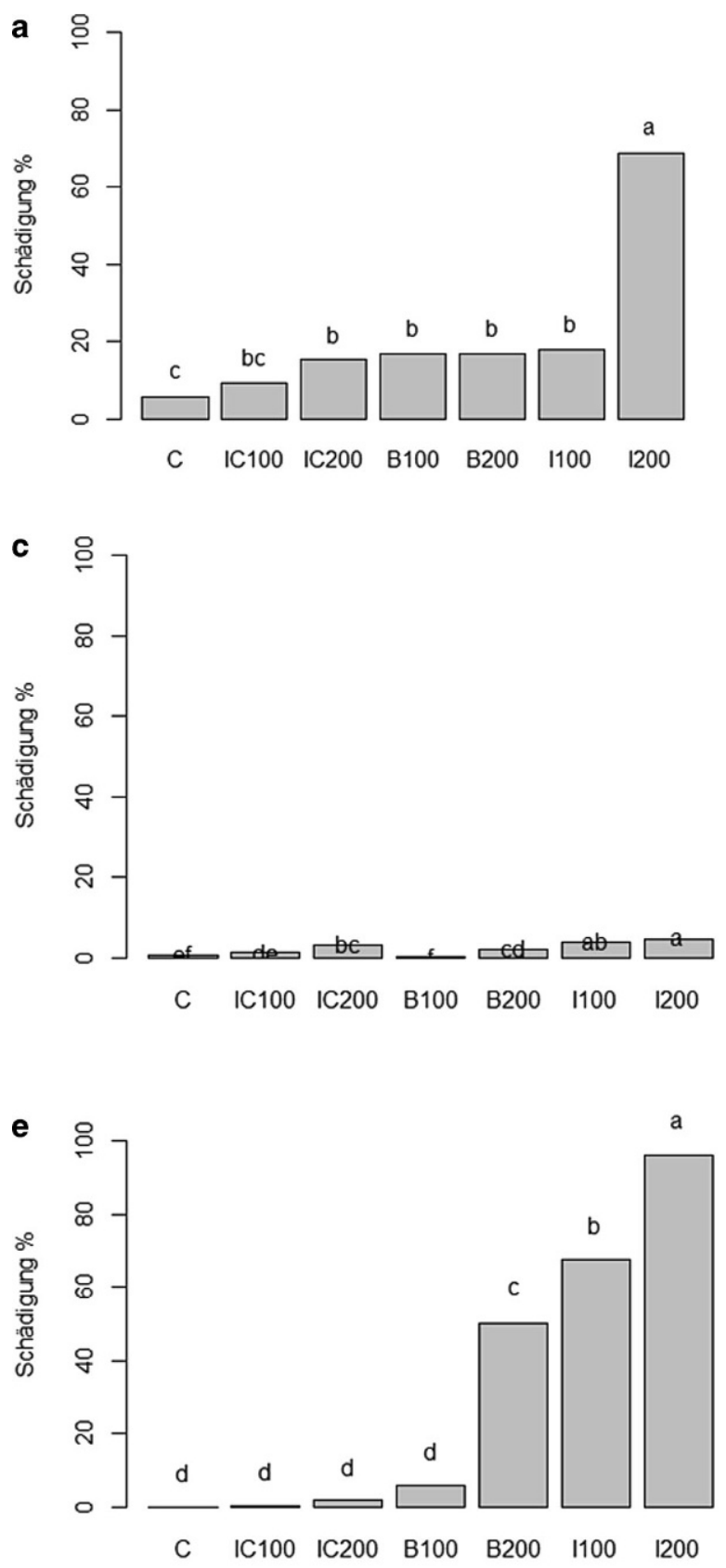

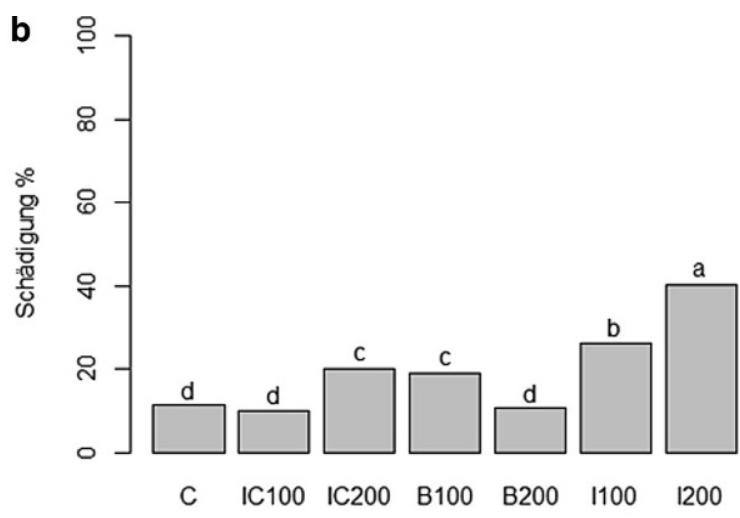

d
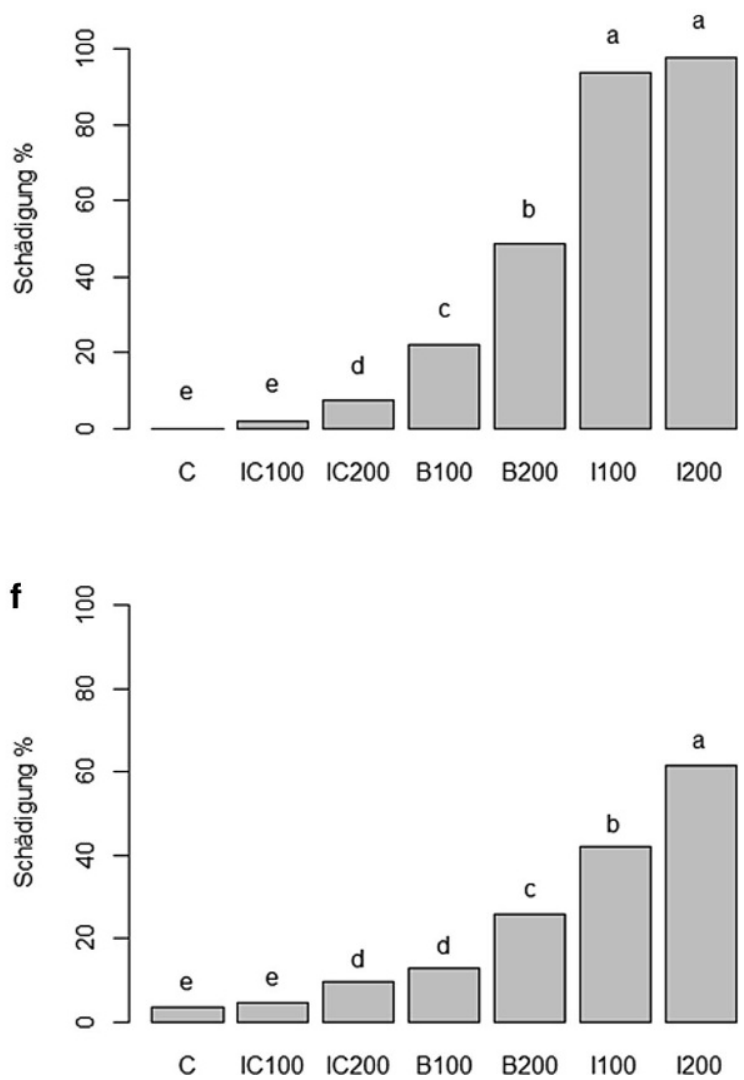

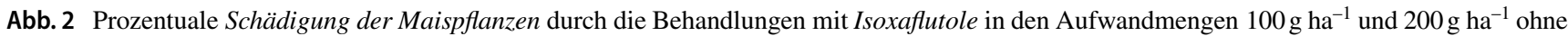
den Safener (I100, I200), mit dem Safener Cyprosulfamid in der Aufwandmenge 2,0 $\mathrm{g} \mathrm{ha}^{-1}$ als Saatgutbehandlung gebeizt (B100, B200) und mit dem Safener Cyprosulfamid in der Aufwandmenge $100 \mathrm{~g} \mathrm{ha}^{-1}$ und $200 \mathrm{~g} \mathrm{ha}^{-1}$ als Co-Formulierung gespritzt (IC 100, IC 200). Die Bonitur erfolgte 28 Tage nach der Behandlung mit Isoxaflutole. Mittelwerte mit gleichem Buchstaben innerhalb einer Grafik sind nicht signifikant voneinander verschieden basierend auf dem Tukey HSD-Test $(p \leq 0,05)$. a Versuch 1, b Versuch 2, c Versuch 3, d Versuch 4, e Versuch 5, f Mittel aller Versuche

\section{Länge der Maissprosse}

Die Reaktion des Längenwachstums des Maissprosses auf die Exposition gegenüber IFT war uneinheitlich. In den meisten Fällen war eine Kürzung des Sprosses zu beobachten, die in den Herbizidvarianten ohne Safener am stärksten und proportional zur Herbiziddosis war. Die Kürzung konnte durch den Safener reduziert werden. In zwei der fünf
Versuche allerdings wuchsen die Sprosse in der Beizvariante nach Anwendung von $100 \mathrm{~g} \mathrm{ha}^{-1}$ IFT länger als in den anderen Versuchsvarianten und übertrafen sogar die Länge unbehandelter Kontrollpflanzen.

Im Mittel der Versuche war das Sprosswachstum des mit der der Co-Formulierung von IFT und CSA behandelten Mais auf dem Niveau der unbehandelten Kontrolle. Die 

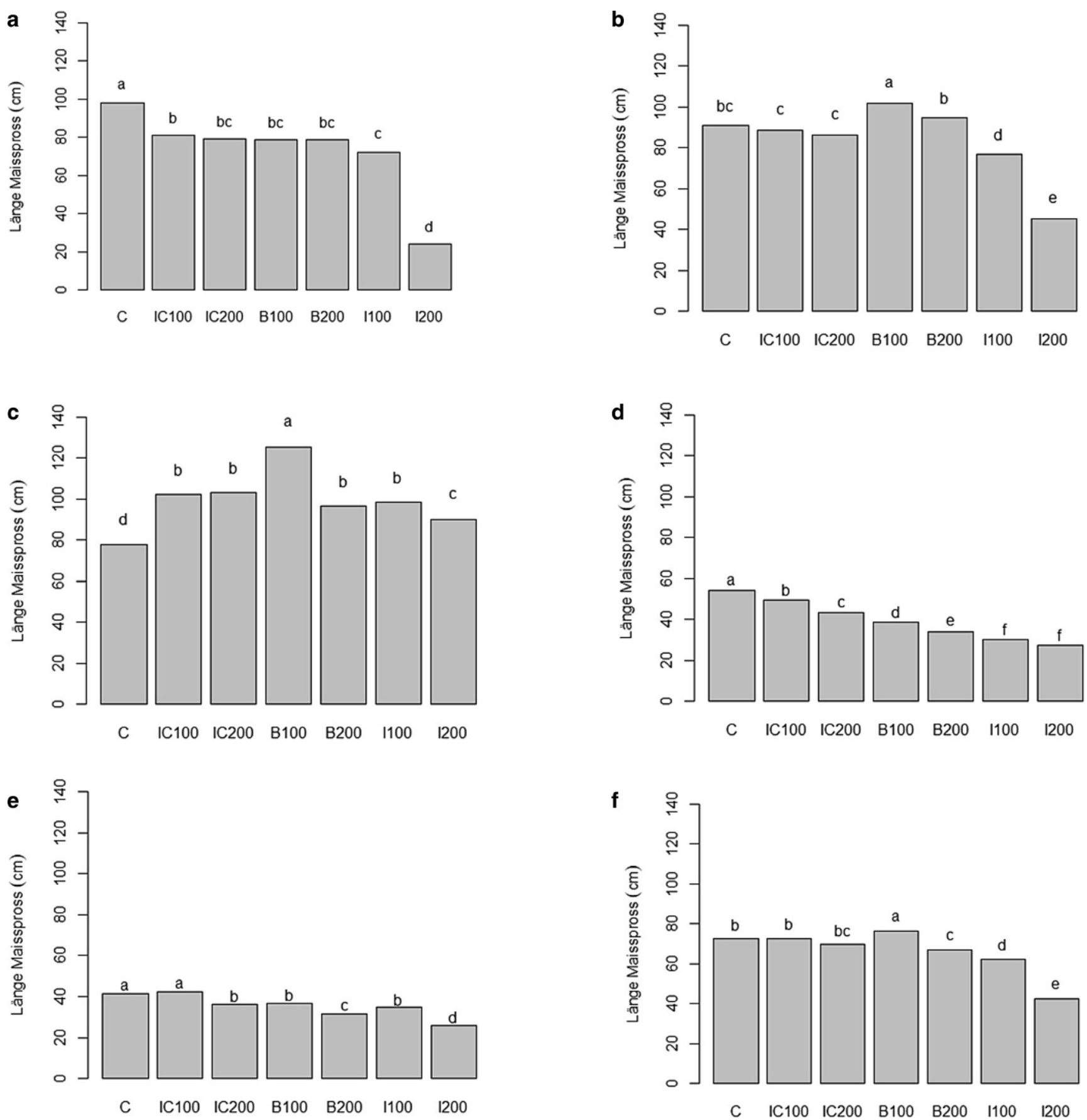

Abb. 3 Sprosslänge der Maispflanzen durch die Behandlungen mit Isoxaflutole in den Aufwandmengen $100 \mathrm{~g} \mathrm{ha}^{-1} \mathrm{und}_{200 \mathrm{~g} \mathrm{ha}}{ }^{-1}$ ohne den Safener (I100, I200), mit dem Safener Cyprosulfamid in der Aufwandmenge 2,0 $\mathrm{g} \mathrm{ha}^{-1}$ als Saatgutbehandlung gebeizt (B100, B200) und mit dem Safener Cyprosulfamid in der Aufwandmenge $100 \mathrm{~g} \mathrm{ha}^{-1}$ und $200 \mathrm{~g} \mathrm{ha}^{-1}$ als Co-Formulierung gespritzt (IC 100, IC 200). Die Bonitur erfolgte 28 Tage nach der Behandlung mit Isoxaflutole. Mittelwerte mit gleichem Buchstaben innerhalb einer Grafik sind nicht signifikant voneinander verschieden basierend auf dem Tukey HSD-Test $(p \leq 0,05)$. a Versuch 1, b Versuch 2, c Versuch 3, d Versuch 4, e Versuch 5, f Mittel aller Versuche

Beizvariante behandelt mit $100 \mathrm{~g} \mathrm{ha}^{-1}$ IFT übertraf diese sogar (Abb. 3).

\section{Trockenmasse des Maisstängels}

Die Behandlungen mit IFT ohne Zusatz des Safeners zeigten die geringste Sprossmassen der Maisstängel (Abb. 4). Mit Zusatz von CSA war der Aufbau von Trockenmasse im orderirdischen Maisspross unter Exposition gegenüber 


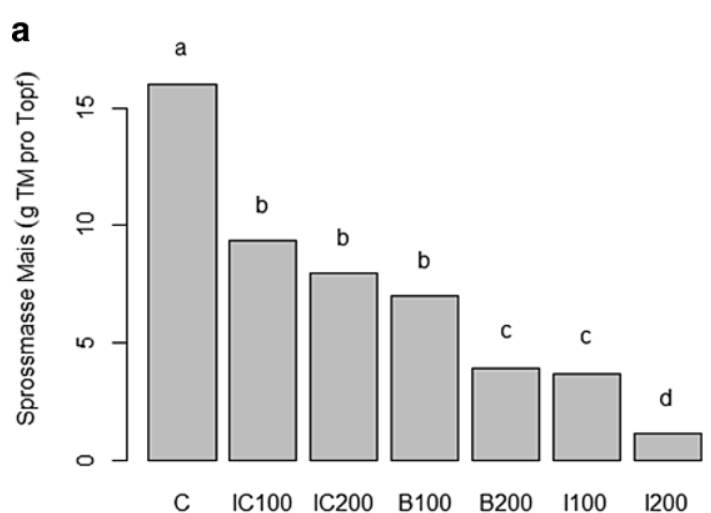

C
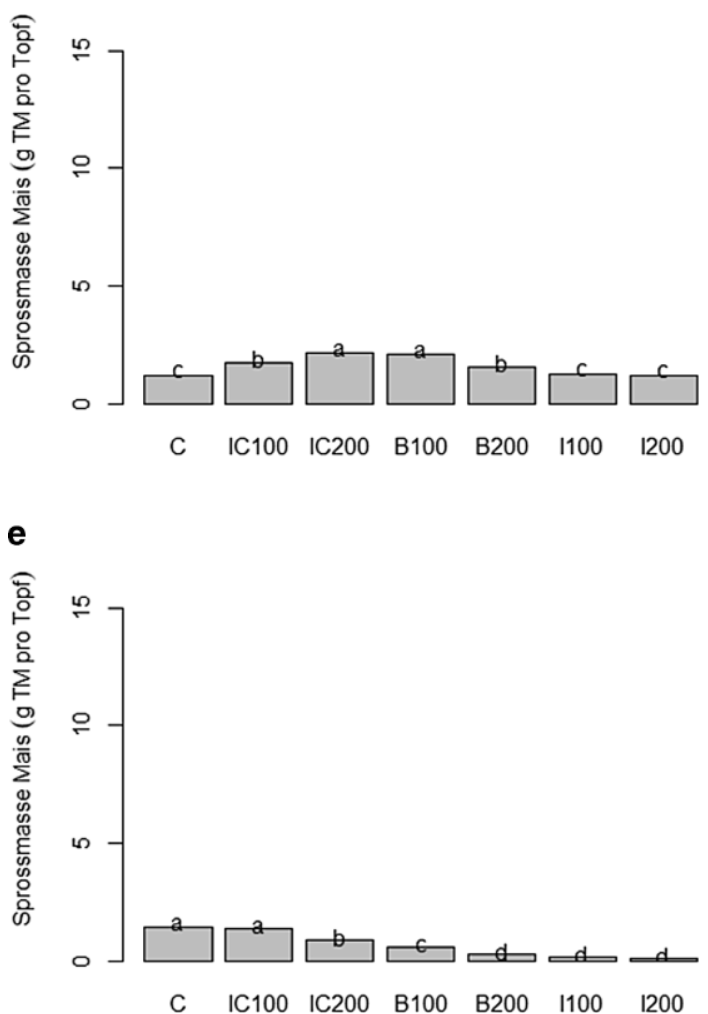

b

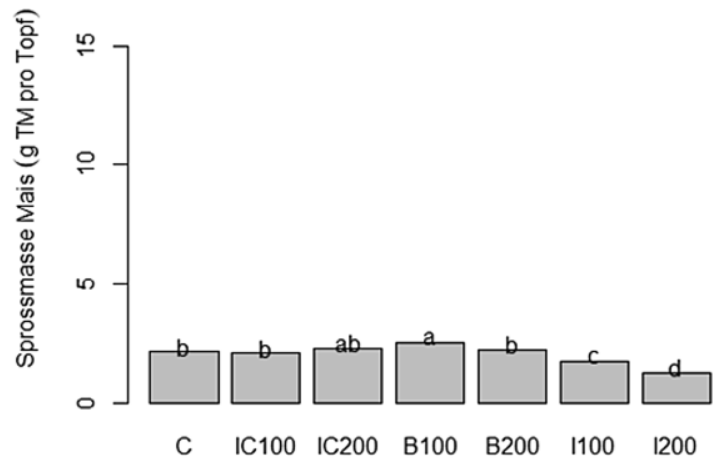

d

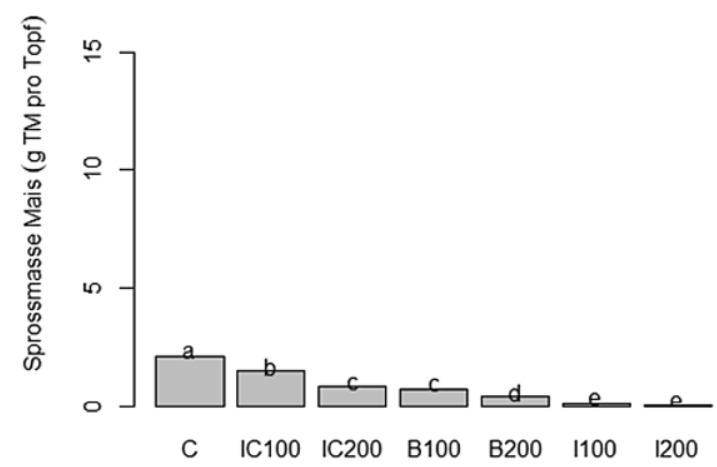

f

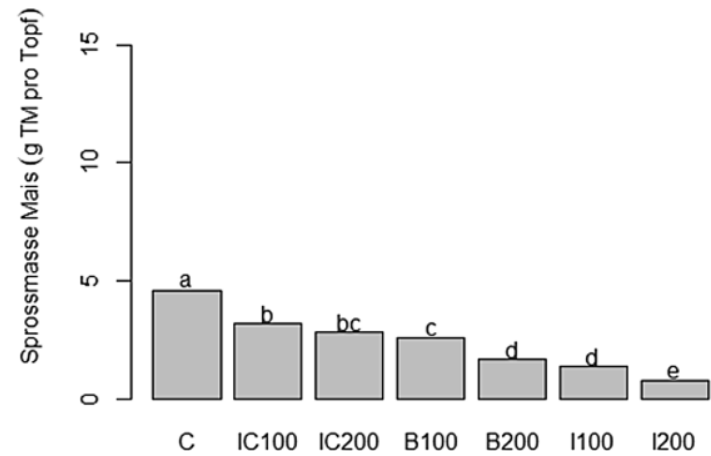

Abb. 4 Trockenmasse der Maissprosse durch die Behandlungen mit Isoxaflutole in den Aufwandmengen $100 \mathrm{~g} \mathrm{ha}^{-1} \mathrm{und}^{200 \mathrm{~g} \text { ha }}{ }^{-1} \mathrm{ohne}$ den Safener (I100, I200), mit dem Safener Cyprosulfamid in der Aufwandmenge 2,0 $\mathrm{g} \mathrm{ha}^{-1}$ als Saatgutbehandlung gebeizt (B100, B200) und mit dem Safener Cyprosulfamid in der Aufwandmenge $100 \mathrm{~g} \mathrm{ha}^{-1}$ und $200 \mathrm{~g} \mathrm{ha}^{-1}$ als Co-Formulierung gespritzt (IC 100, IC 200). Die Bonitur erfolgte 28 Tage nach der Behandlung mit Isoxaflutole. Mittelwerte mit gleichem Buchstaben innerhalb einer Grafik sind nicht signifikant voneinander verschieden basierend auf dem Tukey HSD-Test $(p \leq 0,05)$. a Versuch 1, b Versuch 2, c Versuch 3, d Versuch 4, e Versuch 5, f Mittel aller Versuche

Isoxaflutole uneinheitlich. In zwei der fünf Versuche wurde die Trockenmasse der unbehandelten Kontrolle durch Herbizidbehandlungen in Gegenwart des Safeners übertroffen, unabhängig davon ob der Safener als Beize oder Mischpartner zur Anwendung kam. In drei Versuchen konnte der Safener die Trockenmassebildung nicht auf der Höhe der unbehandelten Kontrolle stabilisieren. Im Mittel aller Versuche konnte der Safener die herbizidbedingte Reduktion der Trockenmassebildung nur teilweise, aber nicht vollständig, verhindern. Die höchste Trockenmasse zeigte die unbehandelte Kontrolle gefolgt von den beiden Co-Formulierungen in beiden Dosierungsstufen und der Beizvariante in Kombination mit $100 \mathrm{~g} \mathrm{ha}^{-1}$ IFT. Deutlich geringer war die Sprosstrockenmasse nach Anwendung der Kombination aus hoher Isoxaflutoldosis und gebeiztem Safener (Abb. 4).

\section{Wirkung gegen Unkräuter}

Die Frage, ob CSA die Wirkung von IFT gegen dikotyle oder monokotyle Unkräuter reduziert, wurde exempla- 
a

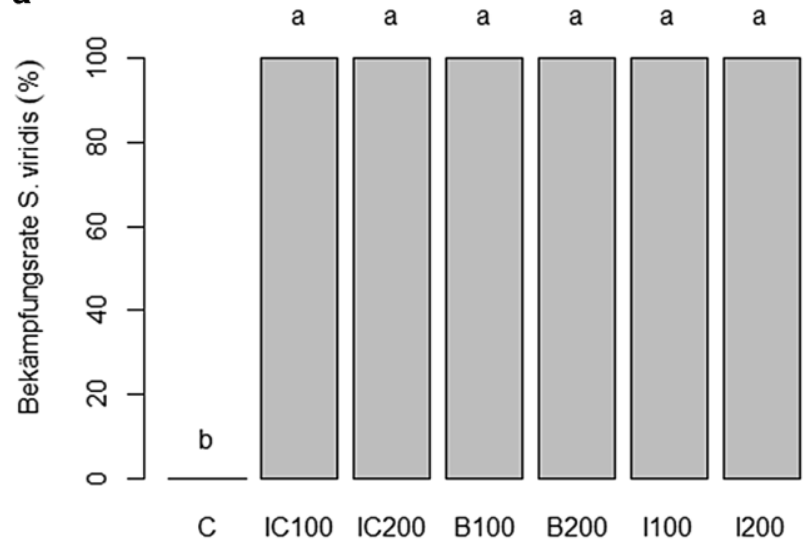

c

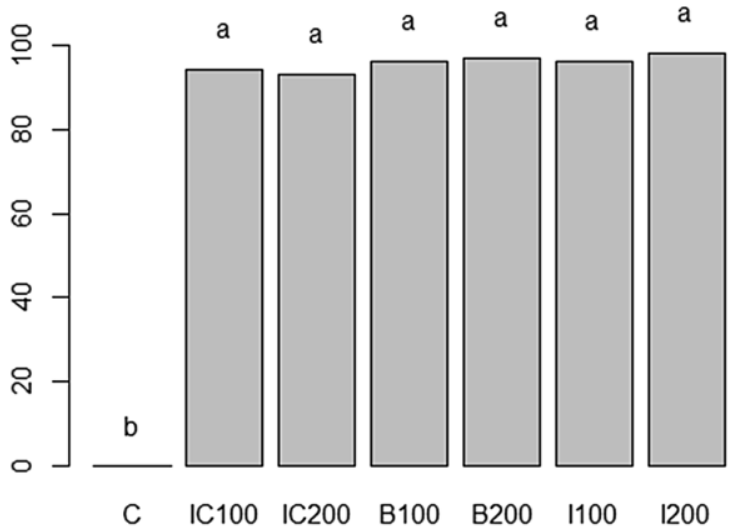

b

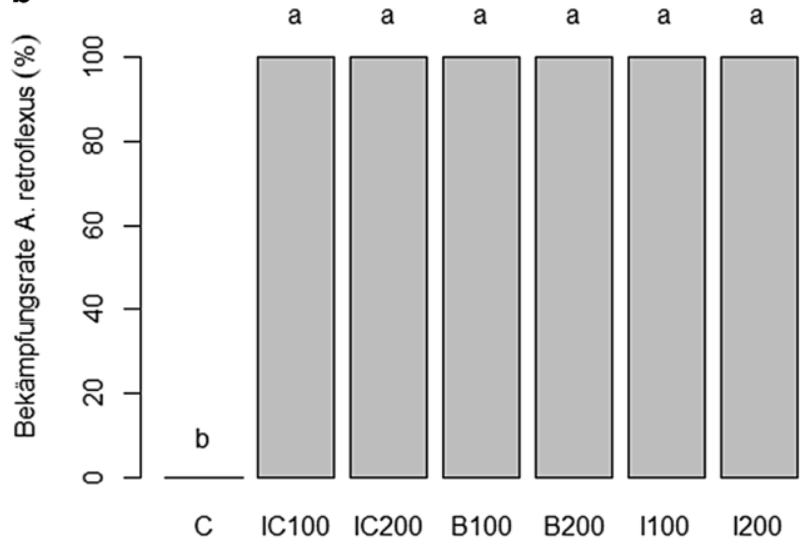

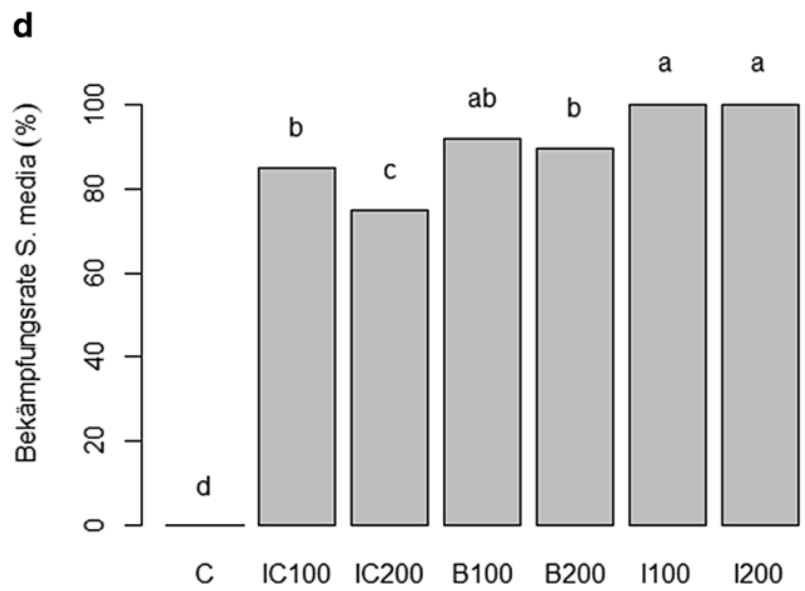

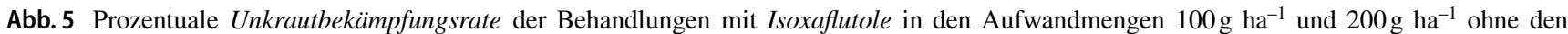
Safener (I100, I200), mit dem Safener Cyprosulfamid in der Aufwandmeng 2,0 $\mathrm{g} \mathrm{ha}^{-1}$ als Saatgutbehandlung gebeizt (B100, B200) und mit dem Safener Cyprosulfamid in der Aufwandmeng $100 \mathrm{~g} \mathrm{ha}^{-1}$ und $200 \mathrm{~g} \mathrm{ha}^{-1}$ als Co-Formulierung gespritzt (IC 100, IC 200) und. Die Bonitur erfolgte 28 Tage nach der Behandlung mit Isoxaflutole. Mittelwerte mit gleichem Buchstaben innerhalb einer Grafik sind nicht signifikant voneinander verschieden basierend auf dem Tukey HSD-Test $(p \leq 0,05)$

risch an Amaranthus retroflexus, Stellaria media, Echinochloa crus-galli und Setaria viridis unterrsucht. Die Bekämpfung von A. retroflexus, E. crus-galli und $S$. viridis gelang in allen Behandlungen zuverlässig und betrug $100 \%$. Nur gegen $S$. media zeigten sich anwendungsabhängige Unterschiede. Diese Unkrautart konnte nur ohne Safener sicher bekämpft werden. Nach Beizung des Safeners war die Wirkung noch gut mit ca. $92 \%$, aber geringer als ohne Safener. Die Wirkung von gleichzeitig angewandtem Safer und Herbizid fiel gegen klar ab mit 79\% (Abb. 5).

\section{Diskussion und Ausblick}

Die Anwendung von IFT auf Mais im Nachauflaufverfahren zeigte ein erhebliches, von der Herbiziddosis abhängiges, Schadensrisiko auf. Die erzielten Ergebnisse belegen, dass der Safener CSA das Ausmaß dieses Schadens erheblich verminderte, unabhängig davon ob der visuell erkennbare
Schaden, die Hemmung des Längenwachstums des oberirdischen Sprosses oder die Bildung von oberirdischer Trockenmasse als Bewertungsparameter gewählt wurde. Die zumindest partielle Kompensation des negativen Effekts des Herbizids auf die Entwicklung von Mais gelang sowohl bei der Anwendung von gleichen Dosierungen von Safener und Herbizid als Nachauflaufspritzung einer entsprechend eingestellten Fertigformulierung als auch nach Vorlage des Safeners als Beize auf dem Saatgut gefolgt von der Nachauflaufanwendung des Herbizids. Bei der praxisüblichen visuellen Bewertung der Verträglichkeit und bei Betrachtung der oberirdischen Trockenmasse war die Schutzwirkung des gebeizten Safeners leicht geringer als die Schutzwirkung des simultan mit dem Herbizid verabreichten Safeners. Die Varianten waren aber nicht signifikant voneinander verschieden. Die schwächere Schutzwirkung des gebeizten Safeners bei der gewählten Dosis könnte eventuell durch eine Dosiserhöhung überwunden werden. Die stabile und ausreichende Schutzwirkung der Anwendung als Fertigformulierung 
belegt, dass die Herbiziddosis und das Herbizid-SafenerVerhältnis im Rahmen der Entwicklung des kommerziellen Produkts erfolgreich optimiert wurden. In jedem Fall muss der Safener so angewandt werden, dass er die Kulturpflanze vor dem Herbizid erreicht und somit vor der Aufnahme des Herbizides durch die Kulturpflanze den Abwehrmechanismus in der Maispflanze anregen kann (Nelson und Penner 2007). Der Safener aus der Co-Formulierung kann über den Boden und das Blatt in die Pflanze eintreten. Im Boden wird CSA vermutlich schneller aufgenommen als IFT, weil CSA einen niedrigeren Absorptionskoeffizienten und eine höhere Wasserlöslichkeit besitzt als IFT (Pallett et al. 2001). Der Wirkstoff IFT muss im Boden und in der Pflanze zuerst in seine aktive herbizide Form umgewandelt werden, was wiederum den Vorteil beinhaltet, dass CSA in der CoFormulierung schneller wirkt als IFT (Pallett et al. 2001). Bei der Saatgutbehandlung mit CSA könnte die Schutzwirkung zumindest bei späten Herbizidapplikation im 4-6Blatt-Stadium verlorengehen. Dies haben Mink et al. (2020) in Feldstudien belegt. Im 2-Blatt-Stadium war die Wirkung von CSA jedoch noch vorhanden.

Die zuverlässige Bekämpfung der im Test verwendeten mono- und dikotylen Unkräuter mit Herbizddosierungen, die für Mais verträglich waren, belegt die Nutzbarkeit von IFT-CSA-Kombinationen zur selektiven Unkrautbekämpfung in Mais. Die durch Cyprosulfamid verursachte Reduktion der Wirkung gegen $S$. media könnte als Hinweis darauf gewertet werden, dass die Wirkung von CSA nicht nur Mais betrifft, sondern erstaunlicherweise auch eine dikotyle Pflanze. Dieses Ergebnis widerspricht zwar den Beobachtungen von Hatzios (1991), wonach Safener bei dikotylen Pflanzen keinen Schutz vor Herbizidschäden hervorrufen. Da sich die Metabolisierung von herbiziden Wirkstoffen in mono- und dikotylen Pflanzen aber ähnelt (Kreuz et al. 1996), besteht die Vermutung, dass die Wirkungsweise von Safenern für alle Pflanzen dieselbe ist (DeRidder et al. 2002). Die beobachtete Verringerung der Wirkung war aber viel zu gering, um von kommerziell relevantem Safening zu sprechen.

Die auch in Gegenwart des Safeners reduzierte oberirdische Trockenmasse weist darauf hin, dass eine Kompensation der Schädigung von Mais über den Beobachtungszeitraum nicht vollständig gelang. Ob unter dem Einfluss des Safeners eine Umverteilung von neu synthetisierter Biomasse zu Ungunsten des Sprosses hin zu anderen Organen stattfand oder ob die durch IFT geschädigte Pflanze sich über einen längeren Zeitraum oder nur unvollständig erholt, bleibt noch zu klären.

Die Schutzwirkung des Safeners reicht aus, um das selektive Anwendungsfenster von IFT vom reinen Vorauflauf bis in den frühen Nachauflauf zu erweitern. Der praktische Nutzen des Wirkstoffs steigt damit deutlich, da seine Anwendungsflexibilität erheblich steigt.
Danksagung Die Autoren danken Alexandra Heyn, Anna Lisa Paulus und Jan-Dilara Aydin für die technische Unterstützung, die Versuchsdurchführung und Messungen in den Topfversuchen.

Funding Open Access funding enabled and organized by Projekt DEAL.

Interessenkonflikt R. Gerhards und H.-J. Santel geben an, dass kein Interessenkonflikt besteht.

Open Access Dieser Artikel wird unter der Creative Commons Namensnennung 4.0 International Lizenz veröffentlicht, welche die Nutzung, Vervielfältigung, Bearbeitung, Verbreitung und Wiedergabe in jeglichem Medium und Format erlaubt, sofern Sie den/die ursprünglichen Autor(en) und die Quelle ordnungsgemäß nennen, einen Link zur Creative Commons Lizenz beifügen und angeben, ob Änderungen vorgenommen wurden.

Die in diesem Artikel enthaltenen Bilder und sonstiges Drittmaterial unterliegen ebenfalls der genannten Creative Commons Lizenz, sofern sich aus der Abbildungslegende nichts anderes ergibt. Sofern das betreffende Material nicht unter der genannten Creative Commons Lizenz steht und die betreffende Handlung nicht nach gesetzlichen Vorschriften erlaubt ist, ist für die oben aufgeführten Weiterverwendungen des Materials die Einwilligung des jeweiligen Rechteinhabers einzuholen.

Weitere Details zur Lizenz entnehmen Sie bitte der Lizenzinformation auf http://creativecommons.org/licenses/by/4.0/deed.de.

\section{Literatur}

Ahrens H, Lange G, Müller T, Rosinger C, Willms L, van Almsick A (2013) 4-Hydroxyphenylpyruvatdioxygenase-Inhibitoren plus Safener: Lösungen für eine moderne und nachhaltige Landwirtschaft. Angew Chem 125:9558-9569

Davies J, Caseley JJ (1999) Herbicide safeners: a review. Pesticide Sci 55:1043-1058

DeRidder BP, Dixon DP, Beussman DJ, Edwards R, Goldsbrough PB (2002) Induction of glutathione S-transferases in Arabidopsis by herbicide safeners. Plant Physiol 130:1497-1505

Gerhards G, Santel HJ (2017) Biologie und Bekämpfung der Unkräuter, 1. Aufl. Fachgebiet Herbologie der Universität Hohenheim, Stuttgart (327 Seiten)

Hamprecht G, Witschel M (2012) Phytoen desaturase inhibitors. In: Kraemer W, Schirmer U, Jeschke P, Witschel M (Hrsg) Herbicides. Modern crop protection compounds, Bd. 1. Wiley-VCH, Weinheim, Germany, S 225-235

Hatzios KK (1991) An overview of the mechanisms of action of herbicide safeners. Z Naturforsch 46:819-827

Hatzios KK, Hoagland RE (Hrsg) (1989) Crop Safeners for herbicides: development, uses, and mechanisms of action. Academic Press, San Diego

Hawkes RT (2012) Hydroxyphenylpyruvate Dioxygenase (HPPD): the target. In: Kraemer W, Schirmer U, Jeschke P, Witschel M (Hrsg) Herbicides. Modern crop protection compounds, Bd. 1. WileyVCH, Weinheim, Germany, S 423-437

Kraehmer H, Laber B, Rosinger C, Schulz A (2014) Herbicides as weed control agents - state of the art. I. Weed control research and safener technology: the path to modern agriculture. Plant Physiol 166:1438-1147

Kreuz K, Tommasini R, Martinoia E (1996) Old enzymes for a new job (herbicide detoxification in plants). Plant Physiol 111:349-353

Mink R, Linn AL, Santel HJ, Gerhards R (2020) Evaluation of herbicide safener treatments using multispectral aerial imagery and hyperspectral ground measured leaf reflectance in maize (Zea mays L.). Pest Manag Sci 76:1856-1865 
Nelson EA, Penner D (2007) Leaching of isoxaflutole and the herbicide safeners R-29148 and furilazole. Weed Technol 21:106-109

Pallett KE, Cramp SM, Little JP, Veerasekaran P, Crudace AJ, Slater AE (2001) Isoxaflutole: the background to its discovery and the basis of its herbicidal properties. Pesticide Manag Sci 57:133-142

Riechers DE, Kreuz K, Zhang Q (2010) Detoxification without intoxication: herbicide safeners activate plant defense gene expression. Plant Physiol 153:3-13

Rosinger C (2014) Herbicide safeners: an overview Bd. 443. JuliusKühn-Archiv, Braunschweig, Germany, S 516-525
Roland Gerhards Prof. Dr. Roland Gerhards leitet seit 2004 das Fachgebiet Herbologie der Universität Hohenheim. Er beschäftigt sich in der Forschung mit Verfahren des Precision Farming, um Unkräuter in Acker- und Gemüsekulturen gezielt zu bekämpfen und den Einsatz von Herbiziden zu vermindern.

Hans-Joachim Santel Herr Dr. Hans-Joachim Santel hat viele Jahre in der Herbizidentwicklung bei Bayer CropScience gearbeitet. Seit 2010 ist er Dozent und freier Wissenschaftler im Fachgebiet Herbologie der Universität Hohenheim. 